\title{
Exploring the mechanisms underlying excitation/inhibition imbalance in human iPSC-derived models of ASD
}

\author{
Lorenza Culotta ${ }^{1,3}$ and Peter Penzes ${ }^{1,2,3^{*}}$ (1)
}

\begin{abstract}
Autism spectrum disorder (ASD) is a range of neurodevelopmental disorders characterized by impaired social interaction and communication, and repetitive or restricted behaviors. ASD subjects exhibit complex genetic and clinical heterogeneity, thus hindering the discovery of pathophysiological mechanisms. Considering that several ASD-risk genes encode proteins involved in the regulation of synaptic plasticity, neuronal excitability, and neuronal connectivity, one hypothesis that has emerged is that ASD arises from a disruption of the neuronal network activity due to perturbation of the synaptic excitation and inhibition (E/I) balance. The development of induced pluripotent stem cell (iPSC) technology and recent advances in neuronal differentiation techniques provide a unique opportunity to model complex neuronal connectivity and to test the E/I hypothesis of ASD in human-based models. Here, we aim to review the latest advances in studying the different cellular and molecular mechanisms contributing to E/l balance using iPSC-based in vitro models of ASD.
\end{abstract}

Keywords: Autism spectrum disorder, Induced pluripotent stem cell, Excitation/inhibition balance

\section{Background}

Autism spectrum disorder (ASD) represents a spectrum of early-onset neurodevelopmental disorders characterized by persistent deficits in social interaction and communication, as well as repetitive patterns of behavior and restricted interests or activities (Diagnostic and Statistical Manual of Mental Disorders [DSM-5]). Current population prevalence of ASD is estimated at $\sim 1.5 \%$ in developed countries around the world and, to date, there are no effective cures $[1,2]$. ASD patients display considerable phenotypic heterogeneity and they often present comorbid neurological and mental conditions, such as epilepsy, intellectual disability (ID), obsessivecompulsive disorder (OCD), and attention-deficit hyperactivity disorder (ADHD) [3].

\footnotetext{
* Correspondence: p-penzes@northwestern.edu

'Department of Physiology, Northwestern University Feinberg School of Medicine, Chicago, IL, USA

${ }^{2}$ Department of Psychiatry and Behavioral Sciences, Northwestern University Feinberg School of Medicine, Chicago, IL, USA

Full list of author information is available at the end of the article
}

ASD can be classified into syndromic and nonsyndromic forms [4-7]. Syndromic ASD accounts for a small percentage of total ASD cases; it typically occurs with a clinical presentation in association with secondary phenotypes and/or dysmorphic features [6]. Most of the syndromic forms of ASD have a known genetic cause, often involving chromosomal abnormalities or mutations in a single gene. On the other hand, non-syndromic ASD accounts for the vast majority of ASD cases and occurs without additional symptoms. In contrast to the syndromic ASD, most of the non-syndromic forms of ASD have unknown genetic etiology [5]. Even though the genetics underlying ASD is complex, ASD is primarily considered a genetic disorder, with family and twins studies suggesting the heritability of ASD to be higher than $80 \%$ [8-12]. Genetic studies have identified several autism-susceptibility (or risk) genes (e.g., SHANK- and $\mathrm{NRXN-family} \mathrm{genes)}$ and copy number variation (CNV) loci (e.g., 16p11.2 deletion and 15q11-q13 duplication), facilitating the molecular diagnosis of ASD cases. Many 
of the identified ASD risk genes are key regulators of synaptic plasticity, with their gene products involved in modulating synaptic strength or density, cell adhesion, chromatin remodeling, transcription, cytoskeleton dynamics and, ultimately, neuronal connectivity [13-15]. In addition to genetic risk factors, several studies suggest that environmental factors may contribute to ASD risk, supporting the hypothesis that ASD could result from the effects of diverse biological and/or psychological factors, like genetic factors, environmental factors, and the interplay between genetic background and environmental factors [16-18]. The environmental factors could be prenatal, perinatal, and postnatal, and some examples include in utero exposure to medications, such as thalidomide and valproate $[19,20]$, parental age of birth, and gestational complications such as diabetes and bleeding $[11,21]$. The speculated existence of interactions between genetic factors and environmental factors suggests that individuals with ASD may react differently to the same environmental stimulus: indeed, studies of animal models have shown that environmental factor, such as hypoxia, oxidative stress, and maternal immune activation, may increase autism risk by interacting with genetic defects in synaptic function [22-24].

One of the proposed etiological mechanisms of ASD is the disruption of the balance between excitation and inhibition (E/I balance) in key cortical and subcortical neuronal circuits [25-30]. E/I balance is a crucial player in the normal development and function of the brain, and different homeostatic and developmental processes appear to be involved in maintaining E/I balance at the level of single cells and large-scale neuronal circuits [26]. At the single neuron level, the balance between excitatory and inhibitory synaptic inputs is critical for information processing, and therefore is highly regulated and structurally organized to allow spatially precise E/I balance across dendritic segments [31,32]. At the network level, $\mathrm{E} / \mathrm{I}$ balance is usually considered as a stable global level of activity within a particular circuit, being the balance of excitation and inhibition important for optimal tuning of the circuits to respond to salient inputs [33, 34]. To date, several studies have described different neurobiological mechanisms contributing to the establishment and rigorous regulation of the E/I balance. Specific factors include intrinsic neuronal excitability, synaptic transmission, and homeostatic synaptic plasticity; at the circuit level, key contributors to E/I balance are the complex interplay between glutamatergic excitatory neurons and GABAergic inhibitory neurons and the development of excitatory and inhibitory synapse, as well as the overall neuronal network excitability [15, 35-37].

A growing body of clinical neuroimaging literature supports the role of E/I imbalance as an etiology of ASD $[28,29]$. Gamma-band electrophysiological activity (30-
$100 \mathrm{~Hz}$ ) is considered to be a functional readout of $\mathrm{E} / \mathrm{I}$ balance within local neural circuits [38]. Studies of gamma-based activity via magnetoencephalography (MEG) and electroencephalography (EEG) have identified alterations in gamma-band activity in ASD patients, especially in relation to auditory-related gamma-band activity; however, despite providing indirect evidence for $\mathrm{E} / \mathrm{I}$ imbalance in ASD, gamma-based activity fundamentally remains a proxy measure $[39,40]$. Magnetic resonance spectroscopy (MRS) has allowed for the direct noninvasive, in vivo estimation of the principal excitatory and inhibitory neurotransmitters, glutamate, and gamma-aminobutyric acid (GABA), and alterations of neurotransmitter levels have been reported within different cortical structures in ASD patients [41-44]. Disruptions in excitation-inhibition ratio have been suggested to correlate with the severity of core ASD symptoms, thus implying that the abnormality in E/I balance is clinically relevant. In particular, glutamate levels in the striatum have been found to negatively correlate to severity of social impairment [42], while a positive correlation has been reported between cortical excitationinhibition ratio and the severity of autistic phenotypes in non-ASD psychiatric subjects [29]. However, these reports are widely inconsistent, possibly due to the different methodologies employed as well as the heterogeneity of subjects between studies, and it is still unknown whether any perturbation in neurotransmitter level reflects a neurotransmission phenotype, rather than a metabolic phenotype [28].

The advent of the induced pluripotent stem cell (iPSC) technology has allowed the generation of personalized human neurons, thus representing a new avenue for the modeling of neurological disorders [45-47]. This model has been made possible through the reprogramming of patient-derived somatic cells, e.g., fibroblasts [46], peripheral blood mononuclear cells [48], or urine cells [49], into pluripotent stem cells via the overexpression of a set of pluripotency-associated transcription factors, Oct4, Sox2, Klf4, and cMyc, named the "Yamanaka factors" [46]. iPSCs have a gene expression profile and pluripotency similar to human embryonic stem cells (hESCs), but with the advantage of easy accessibility and the avoidance of ethical and religious concerns regarding the use of human embryos for research purposes [50, 51]. Given their potential to differentiate into virtually any cell type under appropriate culture conditions, iPSCs could represent a valuable source of disease-relevant cells that were previously inaccessible, such as cardiomyocytes and neurons. As iPSCs can be differentiated into a range of neuronal lineages, iPSC-based disease modeling has greatly contributed to our understanding of neurological diseases, serving as a precious complement to animal models for analyzing neuronal 
development and the consequences of its dysfunctions [46]. This provides an incredibly strong model of disease by recapitulating the entirety of the complex genetic factors that result in patient diagnosis. Therefore, the iPSC approach provides an invaluable tool for investigating the cellular and molecular mechanisms underpinning complex genetic diseases, such as ASD and its related disorders, which include Rett syndrome (RTT), Timothy syndrome (TS), fragile X syndrome (FXS), and PhelanMcDermid syndrome (PMS) [52].

In this review, we will focus on human iPSC-derived in vitro models of ASD and summarize the most recent studies in which the iPSC technology has been exploited to investigate the molecular bases of $\mathrm{E} / \mathrm{I}$ imbalance and to gain further insight into the etiological mechanisms of ASD (Table 1). Being E/I imbalance the focus of this review and being ASD a neurodevelopmental disorder, we decided to include in this review only reports on stem cell-derived neuron in which functional alterations that could impact E/I balance have been reported (Fig. 1). Moreover, given the growing number of ASD risk genes [81], we decided to include only risk genes belonging to SFARI categories 1 and 2, thus excluding genes without strong evidence supporting the gene's relevance to ASD risk.

\section{Main text}

Neuronal excitability and excitatory synaptic transmission In vitro neuronal cultures provide insight into neuronal transmission and network interactions since they retain many of the properties of in vivo networks, such as functional synaptic connectivity and complex patterns of neuronal activity [82, 83]. Considering that defective neuronal networks, as well as reduced functional connectivity, have been widely observed in ASD patients [84], iPSC-derived neuronal cultures provide a valuable method for modeling neuronal activity, allowing the investigation of the intrinsic neuronal excitability, formation of synaptic connections, and overall network activity.

The use of multi-electrode array (MEA) technology has facilitated non-invasive, real-time, multi-point measurement of the activity of cultured neurons and allowed investigating developmental modifications of synaptic connectivity and network activity [85-87]. Therefore, the MEA system is acknowledged as a suitable method for evaluating the plasticity of iPSC-derived neuronal networks and for investigating the molecular basis of the $\mathrm{E} / \mathrm{I}$ imbalance in ASD.

Deneault et al. utilized MEA recordings to evaluate the neuronal network properties in iPSC-derived neurons and to assess the impact of ASD genetic variants on synaptic and intrinsic electrophysiological properties $[53,55]$. Multiple intriguing associations were observed between the genetic variants and the neuronal phenotypes analyzed: one of the most robust findings was a marked hyperexcitability in glutamatergic neurons lacking one copy of CNTN5 or EHMT2 [55], as well as a reduced synaptic activity in $A T R X-$, AFF2-, KCNQ2-, $S C N 2 A-$, and ASTN2-null neurons [53]. Together, these data indicate that ASD-risk genes belonging to different

Table 1 iPSC-based models of ASD discussed in this review

\begin{tabular}{|c|c|c|c|}
\hline Gene & Model type & Phenotype observed & Reference \\
\hline $\begin{array}{l}\text { ATRX, AFF2, KCNQ2, } \\
\text { SCN2A, and ASTN2 }\end{array}$ & Homozygous deletion & Reduced synaptic activity & [53] \\
\hline CACNAIC & ASD-related mutations & Disrupted interneurons migration & [54] \\
\hline CNTN5 and EHMT2 ${ }^{+}$ & Heterozygous deletion & Hyperexcitability. & [55] \\
\hline CNTNAP2 & Heterozygous deletion & Increased neuronal network activity & [56] \\
\hline FMR1 & Heterozygous deletion & Impaired retinoic acid (RA)-dependent homeostatic synaptic plasticity & [57] \\
\hline MECP2 & $\begin{array}{l}\text { Heterozygous deletion or } \\
\text { duplication }\end{array}$ & $\begin{array}{l}\text { Altered synaptic density, altered calcium signaling; altered neuronal firing rate } \\
\text { and synchronization; delayed GABA switch }\end{array}$ & {$[58-61]$} \\
\hline NLGN4 & $\begin{array}{l}\text { Gene overexpression and ASD- } \\
\text { related mutations }\end{array}$ & Increased excitatory synapse density, altered synaptic strength & {$[62,63]$} \\
\hline NRXN1a & $\begin{array}{l}\text { Homozygous and heterozygous } \\
\text { deletion, ASD-related mutations }\end{array}$ & Impaired synaptic strength, altered synaptic calcium signaling & [64-66] \\
\hline SHANK2 & $\begin{array}{l}\text { Heterozygous deletion and ASD- } \\
\text { related mutations }\end{array}$ & Hyperconnectivity, enhanced branching complexity, increased synapse density & [67] \\
\hline SHANK3 & $\begin{array}{l}\text { Heterozygous deletion and ASD- } \\
\text { related mutations }\end{array}$ & $\begin{array}{l}\text { Hypoconnectivity, reduced synaptogenesis, and dendritic arborization; } \\
\text { impaired neuronal excitability and excitatory synaptic transmission; impaired } \\
\text { HCN channels }\end{array}$ & [68-74] \\
\hline $\mathrm{TSC} 1 / 2$ & $\begin{array}{l}\text { Homozygous and heterozygous } \\
\text { deletion }\end{array}$ & $\begin{array}{l}\text { Altered neuronal excitability and activity, altered synchrony (cortical neurons); } \\
\text { hypoexcitability (cerebellar Purkinje cells) }\end{array}$ & {$[75-78]$} \\
\hline Other ASD models & & $\begin{array}{l}\text { Aberrant neuronal maturation, altered neuronal differentiation and synaptic } \\
\text { formation }\end{array}$ & {$[79,80]$} \\
\hline
\end{tabular}




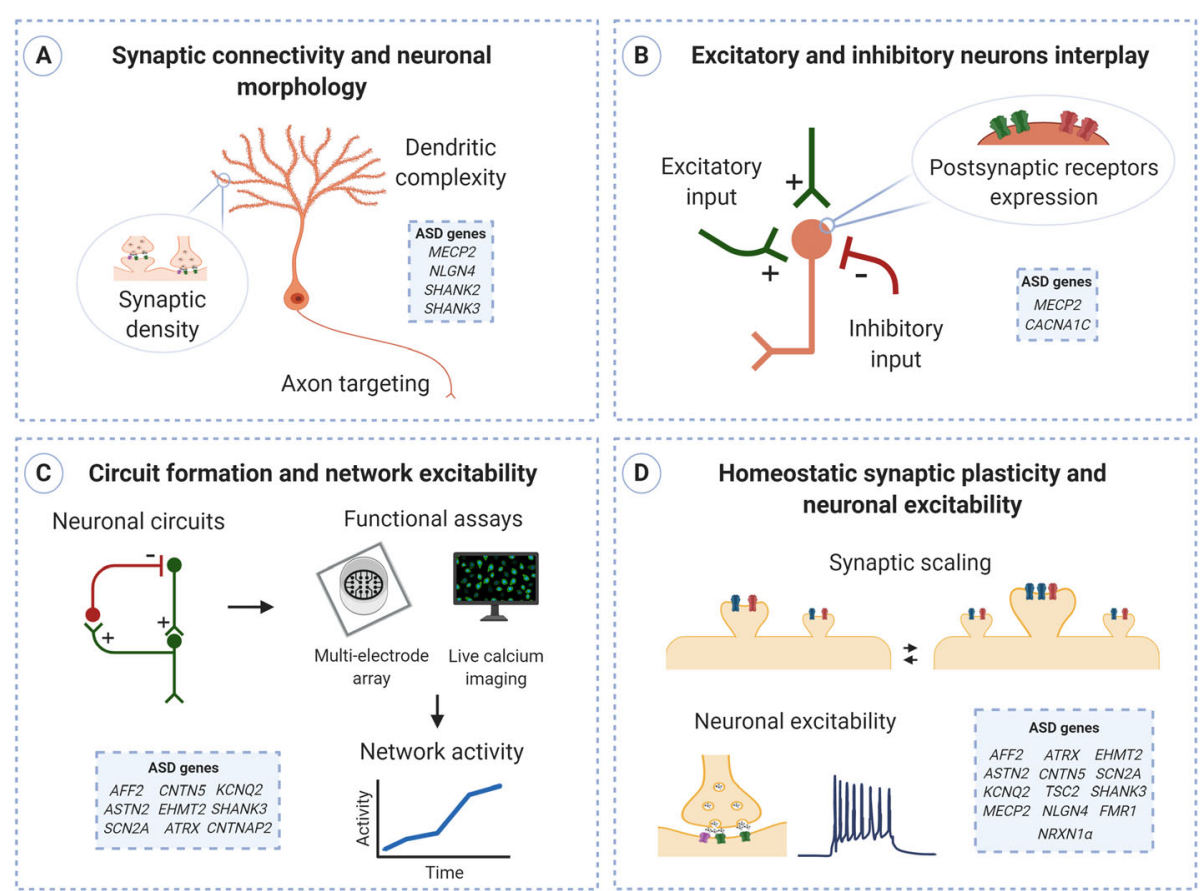

Fig. 1 Neurobiological mechanisms contributing to E/I balance. Schematic illustration of key mechanisms involved in establishing and regulating the balance between excitation and inhibition, highlighting the mechanisms and the ASD-related genes discussed in this review. In the cited reports, the iPSC technology has been exploited to develop human-based platforms in which to investigate the contribution of ASD-related genes to the different processes underlying E/I balance. a Establishment and maintenance of neuronal connectivity and E/I balance require all the salient features of neuronal morphology: the existence of branching dendrites and axons and the presence of neuronal synapses. Alterations in one or more of these features have been reported in several ASD models. $\mathbf{b}$ Excitatory and inhibitory neuron interplay results from the excitatory and inhibitory inputs converging on a neuron, as well as from the level of expression of postsynaptic glutamatergic (green) and GABAergic (red) receptors. c The application of technologies such as multi-electrode arrays and live calcium imaging has facilitated real-time, multi-point measurement of the activity of iPSC-derived neurons and allowed investigating developmental modifications of synaptic connectivity and network activity. $\mathbf{d}$. Synaptic scaling is a form of homeostatic plasticity that operates to modify the global synaptic input (excitability) of a neuron in response to changes in circuit activity. One of the main mechanisms of synaptic scaling is the modification of the density and/or the size of synapses, and multiple electrophysiological techniques are used to analyze synaptic plasticity and neuronal excitability, including miniature excitatory/inhibitory postsynaptic currents

gene ontologies can produce a similar electrophysiological phenotype and reveal common functional phenotypes, such as altered functional connectivity, which could ultimately result in altered excitation/inhibition balance.

\section{SHANK family}

A growing body of literature pinpoints inadequate synaptogenesis and perturbed excitatory synaptic plasticity as key players in the development of dysfunctional neuronal networks and, ultimately, in the E/I imbalance [15, 88-90]. A valid example is represented by mutations in the SHANK family genes, which encode major scaffolding proteins in the excitatory postsynaptic density [9194]. Loss-of-function mutations in the SHANK2 gene have been associated with various neuropsychiatric disorders, such as ASD, intellectual disability, and schizophrenia [95]. In mouse models and primary cultures of neurons, Shank2 has been demonstrated to serve an important role in early neuronal development, neuronal dendrite branching, and basal synaptic transmission [96-99]. In line with these findings, loss-of-function mutations in SHANK2 have been associated with altered connectivity in neurons derived from ASD-affected individuals, as well as CRISPR-Cas9-edited homozygous SHANK2 knockout (KO) neurons [67]. SHANK2 mutant neurons displayed enhanced dendrite branching complexity alongside increased synapse number and spontaneous excitatory postsynaptic currents, thus resulting in strengthened functional neuronal connectivity. Remarkably, the reversal of this phenotype in homozygous $\mathrm{KO}$ neurons via engineering correction of the mutation provides a robust demonstration of the causal role of SHANK2 mutation in determining the functional hyperconnectivity observed.

This phenotype is distinct from the lowered connectivity associated with mutations in SHANK3 [100-102]. SHANK3 gene mutations are strongly linked to ASD, and SHANK3 haploinsufficiency is believed to be the major contributing factor to the 22q13.3 deletion 
syndrome, also known as Phelan-McDermid syndrome (PMS) [103]. Multiple iPSC-based studies sought to assess the functional significance of SHANK3 mutations, using conditional SHANK3-mutant neurons and/or PMS and ASD patient-derived neurons [68-73]. Confirming the crucial role of SHANK3 protein in the regulation of synaptic plasticity, SHANK3 haploinsufficiency resulted in the disruption of synaptogenesis and dendritic complexity, which are critical to ensure proper neuronal connectivity. Indeed, SHANK3-deficient iPSC-derived neurons exhibited impaired dendritic arborization, synaptic density, and intrinsic excitability along with defective excitatory synaptic transmission. Interestingly, Yi et al. have proposed a new role for SHANK3, in addition to having a specifically postsynaptic function [71]. In their work, the authors reported a severe impairment in hyperpolarization-activated cation currents $\left(\mathrm{I}_{\mathrm{h}}\right)$ in SHANK3-deficient hESC-derived neurons. Considering that $\mathrm{I}_{\mathrm{h}}$ currents are mediated by hyperpolarization-activated cyclic nucleotide-gated ( $\mathrm{HCN})$ channels, the authors speculated that SHANK3 might scaffold $\mathrm{HCN}$ channels during neurodevelopment [71]. Notably, HCN-channel mutations are associated with dysfunctional neuronal firing and therefore have been linked to neurological disorders such as epilepsy and impaired learning [104].

Altogether, these findings highlight the relevance of SHANK genes' dosage to neuronal intrinsic excitability and excitatory synaptic transmission, whose disruptions would directly affect E/I balance.

\section{Adhesion molecules}

Defective synaptic transmission has been observed in human neurons carrying mutations in several ASDassociated genes encoding synaptic adhesion molecules. NLGN4 is a member of the neuroligin family and encodes the postsynaptic adhesion molecule Neuroligin-4. Deletions at Xp22.3 encompassing NLGN4 as well as de novo mutations in the NLGN4 gene have been reported in several ASD patients [105]. Neuroligin-4 has been suggested to be an essential element in the regulation of excitatory synaptic transmission. In fact, stem cellderived neurons overexpressing NLGN4 or carrying ASD-related NLGN4 mutations have been reported to have increased excitatory synapse density and altered synaptic strength, thus showing a deleterious effect of NLGN4 dysregulation on synaptic transmission and E/I balance $[62,63,106]$.

Impaired synaptic function has been also reported in human neurons carrying NRXN1 $\alpha$ mutations. NRXN1 $\alpha$ encodes the presynaptic cell-adhesion molecule Neurexin1 ; moreover, mutations and copy number variations of NRXN1 $\alpha$ have been associated with ASD and schizophrenia [107]. iPSC technology has been widely exploited to investigate the functional significance of disease- associated mutation of NRXN1 $\alpha$ in synaptic transmission. Evidence showed that mutations of NRXN1 $\alpha$ severely impaired synaptic transmission by decreasing neurotransmitter release [64]. Controversial results have been obtained when evaluating calcium signaling activity in $N R X N 1 \alpha$ mutant human neurons: in fact, biallelic deletion of NRXN1 $\alpha$ has been associated to decreased calcium dynamics, suggesting decreased intrinsic activity [65], while neurons carrying NRXN1 $\alpha^{+/-}$deletions display increased calcium activity [66]. Although seemingly contradictory, these results show the profound impact of NRXN1 $\alpha$ loss of function on synaptic excitation, further supporting the $\mathrm{E} / \mathrm{I}$ imbalance hypothesis in ASD.

Altered neuronal network activity has also been associated with heterozygous deletion of CNTNAP2. Contactinassociated protein-like 2 (CNTNAP2) is a member of the neurexin family, and gene mutations and common variation in the CNTNAP2 gene have been associated with neurodevelopmental disorders, including ASD [108]. Consistent with reports of disrupted cortical neuronal activity and increased propensity for seizure-like activity in CNTNAP2-null mice [109-112], induced glutamatergic neurons derived from carriers of heterozygous intragenic CNTNAP2 deletions displayed increased neuronal network activity, reflected by the increase in spontaneous spiking activity recorded via MEA, consistent with previous reports [56].

\section{TSC $1 / 2$}

Tuberous sclerosis complex (TSC) provides an example of ASD-associated neuronal excitability abnormalities detected in cortical and non-cortical areas. TSC is a severe multisystem disorder associated with neurologic symptoms including autism, epilepsy, and cognitive disability [113]. TSC is caused by heterozygous loss-offunction mutations in either the TSC1 or TSC2 gene, which are key inhibitory components of the mTORC1 pathway, a regulator of cell development and synaptic homeostasis. Given the implication of enhanced activity of the mTORC1 pathway with ASD, and the high prevalence of ASD in TSC patients [114], several groups have generated iPSC-derived neurons carrying TSC1/2 mutations to investigate the effects of altered TSC-mTORC1 pathway on neuronal development and synaptic physiology. Surprisingly, functional analyses of TSC-mutated neurons led to controversial results. Costa et al. reported reduced excitability in $\mathrm{TSC}^{-/-}$stem cell-derived neurons and a milder phenotype in $T S C 2^{+/-}$neurons, albeit significantly disrupted [75]. On the contrary, Nadadhur et al. observed increased spontaneous calcium event frequency as well as neuronal hyperactivity in TSC patientderived neuronal networks [76]. Winden et al. also confirmed increased neuronal activity in $\mathrm{TSC2}^{-/-}$neurons using multi-electrode arrays, and observed increased neuronal synchrony in both $\mathrm{TSC}^{+/-}$and $\mathrm{TSC}^{-/-}$ 
neurons. Despite opposing effects in activity, these results suggest an abnormality in neuronal connectivity, which may be affected in alternate ways depending on the genetic background of patients [77]. Interestingly, mTORC1 inhibition via rapamycin treatment corrected the excitability deficits both in the case of increased and decreased neuronal activity $[75,77,113]$. These results confirm that deregulated mTORC1 pathway can result in varying phenotypes that, despite seemingly opposing each other, in fact highlight that TSC2 loss of function differentially affects neuronal excitability and, ultimately, E/I regulation.

A high prevalence of ASD in TSC individuals has been associated with cerebellar abnormalities, and dysregulation of the cerebellar cortical development has been suggested as a possible mechanism involved in the development of ASD in TSC patients [115]. In this light, cerebellar Purkinje cells (PC) were derived from TSC patients with ASD, with the aim to characterize the molecular mechanisms of cerebellar dysfunction in ASD and TSC: TSC2-deficient PC have been found to display severe hypoexcitability, reflected by decreased resting membrane potential and lowered spontaneous activity [116].

\section{Homeostatic synaptic plasticity}

Homeostatic synaptic plasticity is a form of neuronal plasticity that operates to adjust the strength of synapses in response to changes in neural activity, thereby refining neuronal connectivity during development and contributing to network stability $[117,118]$. Multiple ASDassociated genes are predicted to influence homeostatic plasticity mechanisms at different levels, as they encode proteins involved in chromatin remodeling, protein synthesis, actin cytoskeleton dynamics, or synaptic transmission [15].

\section{FMR1}

Recent reports indicate that deficits in homeostatic synaptic plasticity might be detrimental for network stability and contribute to cognitive impairment associated with fragile X syndrome (FXS), the most common genetic form of mental retardation with autistic-like behaviors [119]. FXS is caused by the absence of fragile X mental retardation protein (FMRP), an RNA-binding protein involved in dendritic protein synthesis, encoded by the FMR1 gene. Using FXS patient-derived and conditional FMR1 knockout (KO) human embryonic stem (hES) cell lines, Zhang et al. demonstrated that FMRP has an essential role in retinoic acid (RA)-dependent homeostatic synaptic plasticity [57]. Synaptic RA signaling is a key component in the regulation of synaptic strength, and RA is necessary to induce local translation and synaptic scaling [120]. Loss of FMRP expression impaired RA- induced synaptic potentiation, blocking the normal increase in excitatory postsynaptic current (EPSC) amplitude following chronic synaptic silencing. These results demonstrate that FMRP-driven local translation is required postsynaptically for synaptic scaling mediated by RA.

\section{MECP2}

$M E C P 2$ is an example of a relevant protein involved in synaptic plasticity, playing an important role in chromatin remodeling. Females with loss-of-function mutations in the $\mathrm{X}$-linked $M E C P 2$ gene display the progressive neurological disorder Rett syndrome (RTT) [121], whereas boys or girls with duplication of MECP2 develop ASD and ID [122]. It has been demonstrated that MeCP2 plays an essential and cell-autonomous role in homeostatic synaptic plasticity, as neurons lacking $\mathrm{MeCP} 2$ failed to trigger synaptic plasticity and to scale synaptic strength bidirectionally [123, 124]. Neurons generated from RTT patient iPSCs have been widely exploited as a model to recapitulate the early stages of this human neurodevelopmental disease. Marchetto et al. have demonstrated the utility of iPSCs to investigate the functional consequences of MECP2 loss of function. Neurons generated from fibroblasts of RTT patients carrying different $M E C P 2$ mutations exhibited several morphological alterations, including a reduced number of dendritic spines and synapses, and smaller soma size. Moreover, altered intracellular calcium signaling and electrophysiological defects were detected in RTT-derived neuronal networks [58]. Li et al. observed similar synaptic deficits using an isogenic stem cell model of RTT: they reported a significant reduction of action potential rates when recording synchronized events from iPSC-derived neuronal networks [59]. The importance of correct $M E C P 2$ dosage for synaptic plasticity and homeostasis was also investigated in human neurons carrying $M E C P 2$ duplications. Increased glutamatergic synapse number and altered dendritic morphology were observed in $M E C P 2$ duplication neurons, as well as enhanced neuronal network synchronization [60]. These findings confirm a pivotal role of MeCP2 protein dosage in dendritic plasticity and synaptic homeostasis and, ultimately, in neuronal connectivity and electrophysiology.

\section{Neuron differentiation, maturation, and circuit formation Developmental time points}

Many of the genes identified as ASD-risk genes are involved at several time points and in different neural cell types during human cortical development. A commonly used strategy to address this issue is to follow iPSCs during their early neuronal development to examine when and how the earliest ASD-related phenotypes and 
molecular abnormalities arise. Schafer et al. performed time-series transcriptome and cellular phenotype analyses in ASD neural stem cells (NSCs) and identified the NSC stage as a critical developmental period in which dysregulation of specific transcriptional networks arise. The alterations reported in ASD NSCs were a causal factor in later aberrant neuronal maturation of cortical neurons [79]: notably, skipping the NSC stage by direct conversion of iPSC to neurons prevented neuronal ASD phenotypes from manifesting. A similar approach has been used to explore the role of SHANK3 in neuronal development. In a recently published work, Huang et al. performed morphological and functional analyses at different time points during neuronal development in iPSC-derived neurons following SHANK3 knockdown [74]. SHANK3 depletion resulted in reduced growth cone area, neuronal soma size, and neurite complexity at different developmental time points, together with impaired excitatory and inhibitory synaptic transmission, thus confirming in a human-based model the pivotal role of SHANK3 in early stages of neuronal development, in addition to its well characterized role in mature neuronal function.

\section{GABA functional switch}

Given that the complex interplay between glutamatergic excitatory neurons and GABAergic inhibitory neurons is vital to achieving proper cortical neural activity, a considerable effort has been made in the stem cell field to generate neuronal networks with balanced excitatoryinhibitory activities, facilitating studies of disease mechanisms involving E/I imbalance [125, 126]. In this context, the GABA functional switch from excitation to inhibition is critical for the efficacy of GABAergic function. $\mathrm{KCC} 2$, a membrane $\mathrm{K}^{+}-\mathrm{Cl}^{-}$cotransporter and one of the major players in defining the transmembrane $\mathrm{Cl}^{-}$gradient, is a critical downstream gene target of MeCP2. Significant $\mathrm{KCC} 2$ deficits have been reported in a mouse model of Rett syndrome, alterations that were rescued by treatment with recombinant human insulin-like growth factor-1 [127]. Altered expression of KCC2 has been also observed in iPSC-derived neurons from patients with Rett syndrome [61]. In fact, the lack of MeCP2 in human Rett neurons led to deficient KCC2 expression, and therefore a delayed GABA function switch. As proper inhibitory input is crucial to the development of normal neuronal circuitry, this novel pathway provides insight into the potential mechanisms of Rett syndrome.

\section{Brain organoids}

The advent of 3D cultures, the most recent stem cellbased model for investigating human brain development and disorders, has provided particular strength to the generation of informative neuronal networks [128, 129]. 3D neuronal cultures, also known as organoids or spheroids, have the potential to recapitulate the structural and functional complexity of the human brain with greater complexity than 2D models. One of the major advantages of the 3D technology is the diversity of cell types present in the organoids, including, but not restricted to, glutamatergic neurons, GABAergic neurons, astrocytes, and oligodendrocytes $[130,131]$. Consequently, organoids represent an invaluable and versatile tool to investigate neuronal differentiation and neural circuit formation, essential features underlying network-level E/ I balance. For instance, Mariani et al. demonstrated that telencephalic organoids derived from ASD patients present altered neuronal differentiation and synaptic formation, alongside enhanced GABAergic differentiation, which together resulted in an E/I imbalance [80]. Notably, the overproduction of GABAergic neurons was ascribed to overexpression of the transcription factor FOXG1: taken together with the alterations linked to $\mathrm{MeCP} 2$, these results reinforce the importance of proper gene expression in ASD.

iPSC-derived spheroids have allowed the assembly of the different neuronal circuits to be modeled, in a manner similar to that which occurs in human cerebral cortex formation [132]. Therefore, they have provided a precious tool to investigate the molecular processes that can lead to an imbalance of cortical excitation and inhibition. Timothy syndrome (TS) is a severe ASD-related neurodevelopmental disorder caused by mutations in the CACNA1C gene, encoding the CaV1.2 calcium channel [133]. In a model of TS, neural spheroids were generated to recapitulate the assembling of circuits involving both glutamatergic neurons and GABAergic interneurons and exploited to investigate migration of interneurons and their functional integration into human-derived forebrain spheroids [54]. TS-derived interneurons showed inefficient migration, resulting from altered L-type calcium channel (LTCC) activity, which plays a critical role in interneuron migration: in fact, the TS migratory phenotype was rescued by application of the LTCC blocker nimodipine [54]. These results suggest the presence of dysfunctional cortical development and E/I balance in TS.

One of the latest advances in the modeling of early neurodevelopment is the use of iPSC-derived cortical organoids to model neural oscillations [83]. Synchronous oscillations are periodical and regular network activity events that emerge early in development and are dependent on balanced glutamatergic and GABAergic signaling [134]. As E/I balance is crucial for the coordination of neuronal oscillatory activity, and is one of the leading hypotheses in the etiology of ASD [25], it is not surprising that impairments in neuronal oscillatory 
activity and synchrony have been proposed to be correlated with social behavior deficits in ASD [135]. Thus, modeling organoid neural oscillations may allow the reproduction of a central hallmark of the in vivo brain: a highly synchronous and stereotypical network activity. Such an advancement would open new avenues in characterizing normal and dysfunctional activity in brain networks during early neurodevelopment and within ASD.

\section{Conclusions}

The complex genetic etiologies and clinical phenotypes observed in ASD patients hinder the research of the pathophysiological mechanisms underpinning ASD and the quest for pharmacological treatments. Although animal models have deepened our understanding of disease mechanisms and shed light on possible therapeutic approaches, there has been little translational benefit for human patients. iPSC technology allows the generation of patient-specific neuronal cells, thus representing a powerful platform for investigating disease mechanisms and developing targeted therapies. Even though much work remains to be done to overcome the technical limitations of iPSC-based models (e.g., experimental variability, cellular heterogeneity, or lack of some brain cell types), their study has provided invaluable insight into the molecular and cellular mechanisms underlying ASD. In particular, a growing body of literature has provided evidence of altered balance between excitation and inhibition in human-based models of ASD. These have been linked to disruptions in different neuronal mechanisms contributing to the generation and regulation of $\mathrm{E} / \mathrm{I}$ balance, such as homeostatic plasticity, synaptic transmission, and neuronal excitability. Furthermore, the recent advent of brain organoids has represented a great advance in modeling of ASD, providing a more valuable model of brain development in which to assess E/I imbalance. Indeed, organoids recapitulate several morphological and functional features of the developing human brain, and have the potential to be a more predictive drug screening platform. These advances support the study of iPSC-derived neuronal systems as key in unlocking pathogenic mechanisms and potential treatment avenues in ASD.

\footnotetext{
Abbreviations

ADHD: Attention-deficit hyperactivity disorder; ASD: Autism spectrum disorder; CNV: Copy number variation; E/l: Excitation/inhibition; EEG: Electroencephalography; EPSC: Excitatory postsynaptic current; FXS: Fragile X syndrome; GABA: Gamma-aminobutyric acid; HCN: Hyperpolarization-activated cyclic nucleotide-gated; ID: Intellectual disability; iPSC: Induced pluripotent stem cell; LTCC: L-type calcium channel; KO: Knockout; MEA: Multi-electrode array; MEG: Magnetoencephalography; MRS: Magnetic resonance spectroscopy; NSC: Neural stem cell; OCD: Obsessive-compulsive disorder; PC: Purkinje cells; PMS: PhelanMcDermid syndrome; RA: Retinoic acid; RTT: Rett syndrome; TS: Timothy syndrome; TSC: Tuberous sclerosis complex
}

\section{Acknowledgements}

We thank Dr. Euan Parnell for the discussion and help with editing the manuscript. Figure was created with Biorender.com (https://biorender.com/).

\section{Authors' contributions}

LC performed the literature review, wrote the manuscript, and prepared the figure and the table. PP supervised the writing of the manuscript, critically reviewed, and approved the final manuscript for publication. Both authors read and approved the final manuscript.

\section{Funding}

This work was supported by a National Institute of Health grant. Grant ref.: 5RO1MH097216.

\section{Availability of data and materials \\ Not applicable.}

Ethics approval and consent to participate

Not applicable.

Consent for publication

Not applicable.

\section{Competing interests}

The authors declare no competing interests.

\section{Author details}

${ }^{1}$ Department of Physiology, Northwestern University Feinberg School of Medicine, Chicago, IL, USA. ${ }^{2}$ Department of Psychiatry and Behavioral Sciences, Northwestern University Feinberg School of Medicine, Chicago, IL, USA. ${ }^{3}$ Center for Autism and Neurodevelopment, Northwestern University, Chicago, IL, USA.

Received: 11 February 2020 Accepted: 21 April 2020

Published online: 11 May 2020

\section{References}

1. Baio J, et al. Prevalence of autism spectrum disorder among children aged 8 years - autism and developmental disabilities monitoring network, 11 sites, United States, 2014. MMWR Surveill Summ. 2018;67(6):1-23.

2. Lyall $K$, et al. The changing epidemiology of autism spectrum disorders. Annu Rev Public Health. 2017;38:81-102.

3. Huguet G, Ey E, Bourgeron T. The genetic landscapes of autism spectrum disorders. Annu Rev Genomics Hum Genet. 2013;14:191-213.

4. Fernandez BA, Scherer SW. Syndromic autism spectrum disorders: moving from a clinically defined to a molecularly defined approach. Dialogues Clin Neurosci. 2017;19(4):353-71.

5. Bury LA, Wynshaw-Boris A. Modeling non-syndromic autism with humaninduced pluripotent stem cells. Neuropsychopharmacology. 2018;43(1):219-20.

6. Sztainberg $Y$, Zoghbi HY. Lessons learned from studying syndromic autism spectrum disorders. Nat Neurosci. 2016;19(11):1408-17.

7. de la Torre-Ubieta $L$, et al. Advancing the understanding of autism disease mechanisms through genetics. Nat Med. 2016;22(4):345-61.

8. Sandin S, et al. The heritability of autism spectrum disorder. JAMA. 2017; 318(12):1182-4.

9. Sullivan PF, Geschwind DH. Defining the genetic, genomic, cellular, and diagnostic architectures of psychiatric disorders. Cell. 2019;177(1):162-83.

10. Lundstrom $\mathrm{S}$, et al. Autism spectrum disorders and autistic like traits: similar etiology in the extreme end and the normal variation. Arch Gen Psychiatry. 2012;69(1):46-52.

11. Hallmayer J, et al. Genetic heritability and shared environmental factors among twin pairs with autism. Arch Gen Psychiatry. 2011;68(11):1095-102.

12. Colvert $\mathrm{E}$, et al. Heritability of autism spectrum disorder in a UK populationbased twin sample. JAMA Psychiatry. 2015;72(5):415-23.

13. O'Roak BJ, et al. Sporadic autism exomes reveal a highly interconnected protein network of de novo mutations. Nature. 2012;485(7397):246-50.

14. Iossifov I, et al. The contribution of de novo coding mutations to autism spectrum disorder. Nature. 2014;515(7526):216-21.

15. Bourgeron T. From the genetic architecture to synaptic plasticity in autism spectrum disorder. Nat Rev Neurosci. 2015;16(9):551-63. 
16. Dietert RR, Dietert JM, Dewitt JC. Environmental risk factors for autism Emerg Health Threats J. 2011;4:7111.

17. Tordjman $S$, et al. Gene $x$ Environment interactions in autism spectrum disorders: role of epigenetic mechanisms. Front Psychiatry. 2014;5:53.

18. Chaste P, Leboyer M. Autism risk factors: genes, environment, and geneenvironment interactions. Dialogues Clin Neurosci. 2012;14(3):281-92.

19. Bromley RL, et al. Autism spectrum disorders following in utero exposure to antiepileptic drugs. Neurology. 2008;71(23):1923-4.

20. Miyazaki K, Narita N, Narita M. Maternal administration of thalidomide or valproic acid causes abnormal serotonergic neurons in the offspring: implication for pathogenesis of autism. Int J Dev Neurosci. 2005;23(2-3): 287-97.

21. Gardener H, Spiegelman D, Buka SL. Perinatal and neonatal risk factors for autism: a comprehensive meta-analysis. Pediatrics. 2011;128(2):344-55.

22. Fischer $\mathrm{M}$, et al. Enhanced hypoxia susceptibility in hippocampal slices from a mouse model of rett syndrome. J Neurophysiol. 2009;101(2): 1016-32.

23. Hunter JW, et al. Neuroligin-deficient mutants of $C$. elegans have sensory processing deficits and are hypersensitive to oxidative stress and mercury toxicity. Dis Model Mech. 2010;3(5-6):366-76.

24. Ehninger D, et al. Gestational immune activation and Tsc2 haploinsufficiency cooperate to disrupt fetal survival and may perturb social behavior in adult mice. Mol Psychiatry. 2012;17(1):62-70.

25. Rubenstein $\mathrm{JL}$, Merzenich MM. Model of autism: increased ratio of excitation/inhibition in key neural systems. Genes Brain Behav. 2003;2(5): 255-67.

26. Sohal VS, Rubenstein JLR. Excitation-inhibition balance as a framework for investigating mechanisms in neuropsychiatric disorders. Mol Psychiatry. 2019;24(9):1248-57.

27. Howell BW, Smith KM. Synaptic structural protein dysfunction leads to altered excitation inhibition ratios in models of autism spectrum disorder. Pharmacol Res. 2019;139:207-14.

28. Port RG, Oberman LM, Roberts TP. Revisiting the excitation/inhibition imbalance hypothesis of ASD through a clinical lens. Br J Radiol. 2019; 92(1101):20180944.

29. Oliveira B, et al. Excitation-inhibition dysbalance as predictor of autistic phenotypes. J Psychiatr Res. 2018;104:96-9.

30. Bozzi Y, Provenzano G, Casarosa S. Neurobiological bases of autism-epilepsy comorbidity: a focus on excitation/inhibition imbalance. Eur J Neurosci. 2018:47(6):534-48.

31. Jain $\mathrm{V}$, et al. The functional organization of excitation and inhibition in the dendrites of mouse direction-selective ganglion cells. Elife. 2020;9.

32. lascone DM, et al. Whole-neuron synaptic mapping reveals spatially precise excitatory/inhibitory balance limiting dendritic and somatic spiking. Neuron. 2020.

33. Shew $W L$, et al. Information capacity and transmission are maximized in balanced cortical networks with neuronal avalanches. J Neurosci. 2011;31(1): 55-63.

34. Canitano R, Pallagrosi M. Autism spectrum disorders and schizophrenia spectrum disorders: excitation/inhibition imbalance and developmental trajectories. Front Psychiatry. 2017;8:69.

35. Geschwind DH, Levitt P. Autism spectrum disorders: developmental disconnection syndromes. Curr Opin Neurobiol. 2007;17(1):103-11.

36. Gao R, Penzes P. Common mechanisms of excitatory and inhibitory imbalance in schizophrenia and autism spectrum disorders. Curr Mol Med. 2015;15(2):146-67.

37. Lee E, Lee J, Kim E. Excitation/inhibition imbalance in animal models of autism spectrum disorders. Biol Psychiatry. 2017:81(10):838-47.

38. Whittington MA, et al. Inhibition-based rhythms: experimental and mathematical observations on network dynamics. Int J Psychophysiol. 2000; 38(3):315-36.

39. Port RG, et al. Maturation of auditory neural processes in autism spectrum disorder - a longitudinal MEG study. Neuroimage Clin. 2016;11:566-77.

40. Edgar JC, et al. Neuromagnetic oscillations predict evoked-response latency delays and core language deficits in autism spectrum disorders. J Autism Dev Disord. 2015:45(2):395-405.

41. Mescher M, et al. Simultaneous in vivo spectral editing and water suppression. NMR Biomed. 1998;11(6):266-72.

42. Horder J, et al. Glutamate and GABA in autism spectrum disorder-a translational magnetic resonance spectroscopy study in man and rodent models. Transl Psychiatry. 2018;8(1):106.
43. Brown MS, et al. Increased glutamate concentration in the auditory cortex of persons with autism and first-degree relatives: a (1)H-MRS study. Autism Res. 2013;6(1):1-10.

44. Kubas B, et al. Metabolite alterations in autistic children: a $1 \mathrm{H} \mathrm{MR}$ spectroscopy study. Adv Med Sci. 2012;57(1):152-6.

45. Ross PJ, Ellis J. Modeling complex neuropsychiatric disease with induced pluripotent stem cells. F1000 Biol Rep. 2010;2:84.

46. Takahashi $\mathrm{K}$, et al. Induction of pluripotent stem cells from adult human fibroblasts by defined factors. Cell. 2007;131(5):861-72.

47. Shi Y, et al. Induced pluripotent stem cell technology: a decade of progress. Nat Rev Drug Discov. 2017;16(2):115-30

48. Staerk J, et al. Reprogramming of human peripheral blood cells to induced pluripotent stem cells. Cell Stem Cell. 2010;7(1):20-4.

49. Zhou T, et al. Generation of human induced pluripotent stem cells from urine samples. Nat Protoc. 2012;7(12):2080-9.

50. Bilic J, Izpisua Belmonte JC. Concise review: induced pluripotent stem cells versus embryonic stem cells: close enough or yet too far apart? Stem Cells. 2012;30(1):33-41.

51. Halevy T, Urbach A. Comparing ESC and iPSC-based models for human genetic disorders. J Clin Med. 2014;3(4):1146-62.

52. Vitrac A, Cloez-Tayarani I. Induced pluripotent stem cells as a tool to study brain circuits in autism-related disorders. Stem Cell Res Ther. 2018;9(1):226.

53. Deneault $\mathrm{E}$, et al. Complete disruption of autism-susceptibility genes by gene editing predominantly reduces functional connectivity of isogenic human neurons. Stem Cell Reports. 2018;11(5):1211-25.

54. Birey F, et al. Assembly of functionally integrated human forebrain spheroids. Nature. 2017;545(7652):54-9.

55. Deneault E, et al. CNTN5(-)(/+)or EHMT2(-)(/+)human iPSC-derived neurons from individuals with autism develop hyperactive neuronal networks. Elife. 2019;8.

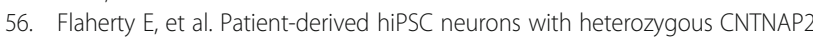
deletions display altered neuronal gene expression and network activity. NPJ Schizophr. 2017;3(1):35.

57. Zhang $Z$, et al. The fragile $X$ mutation impairs homeostatic plasticity in human neurons by blocking synaptic retinoic acid signaling. Sci Transl Med. 2018;10(452).

58. Marchetto MC, et al. A model for neural development and treatment of Rett syndrome using human induced pluripotent stem cells. Cell. 2010;143(4): $527-39$

59. Li Y, et al. Global transcriptional and translational repression in humanembryonic-stem-cell-derived Rett syndrome neurons. Cell Stem Cell. 2013; 13(4):446-58.

60. Nageshappa $\mathrm{S}$, et al. Altered neuronal network and rescue in a human MECP2 duplication model. Mol Psychiatry. 2016;21(2):178-88.

61. Tang $X$, et al. KCC2 rescues functional deficits in human neurons derived from patients with Rett syndrome. Proc Natl Acad Sci U S A. 2016;113(3): 751-6.

62. Marro SG, et al. Neuroligin-4 regulates excitatory synaptic transmission in human neurons. Neuron. 2019;103(4):617-26 e6.

63. Kim JE, et al. Investigating synapse formation and function using human pluripotent stem cell-derived neurons. Proc Natl Acad Sci U S A. 2011; 108(7):3005-10.

64. Pak $C$, et al. Human neuropsychiatric disease modeling using conditional deletion reveals synaptic transmission defects caused by heterozygous mutations in NRXN1. Cell Stem Cell. 2015;17(3):316-28.

65. Lam M, et al. Single cell analysis of autism patient with bi-allelic NRXN1alpha deletion reveals skewed fate choice in neural progenitors and impaired neuronal functionality. Exp Cell Res. 2019;383(1):111469.

66. Avazzadeh $\mathrm{S}$, et al. Increased $\mathrm{Ca}(2+)$ signaling in NRXN1alpha (+/-) neurons derived from ASD induced pluripotent stem cells. Mol Autism. 2019;10:52.

67. Zaslavsky K, et al. SHANK2 mutations associated with autism spectrum disorder cause hyperconnectivity of human neurons. Nat Neurosci. 2019; 22(4):556-64.

68. Shcheglovitov A, et al. SHANK3 and IGF1 restore synaptic deficits in neurons from 22q13 deletion syndrome patients. Nature. 2013;503(7475):267-71.

69. Bidinosti M, et al. CLK2 inhibition ameliorates autistic features associated with SHANK3 deficiency. Science. 2016;351(6278):1199-203.

70. Darville $\mathrm{H}$, et al. Human pluripotent stem cell-derived cortical neurons for high throughput medication screening in autism: a proof of concept study in SHANK3 haploinsufficiency syndrome. EBioMedicine. 2016;9:293-305. 
71. Yi F, et al. Autism-associated SHANK3 haploinsufficiency causes Ih channelopathy in human neurons. Sci. 2016;352(6286):aaf2669.

72. Kathuria A, et al. Stem cell-derived neurons from autistic individuals with SHANK3 mutation show morphogenetic abnormalities during early development. Mol Psychiatry. 2018;23(3):735-46.

73. Gouder $L$, et al. Altered spinogenesis in iPSC-derived cortical neurons from patients with autism carrying de novo SHANK3 mutations. Sci Rep. 2019; 9(1):94.

74. Huang $G$, et al. Uncovering the functional link between SHANK3 deletions and deficiency in neurodevelopment using iPSC-derived human neurons. Front Neuroanat. 2019;13:23.

75. Costa $\mathrm{V}$, et al. mTORC1 inhibition corrects neurodevelopmental and synaptic alterations in a human stem cell model of tuberous sclerosis. Cell Rep. 2016;15(1):86-95.

76. Nadadhur AG, et al. Neuron-glia interactions increase neuronal phenotypes in tuberous sclerosis complex patient iPSC-derived models. Stem Cell Reports. 2019:12(1):42-56.

77. Winden KD, et al. Biallelic mutations in TSC2 lead to abnormalities associated with cortical tubers in human iPSC-derived neurons. J Neurosci. 2019;39(47):9294-305.

78. Henry FE, et al. A unique homeostatic signaling pathway links synaptic inactivity to postsynaptic mTORC1. J Neurosci. 2018;38(9):2207-25.

79. Schafer ST, et al. Pathological priming causes developmental gene network heterochronicity in autistic subject-derived neurons. Nat Neurosci. 2019; 22(2):243-55.

80. Mariani J, et al. FOXG1-dependent dysregulation of GABA/glutamate neuron differentiation in autism spectrum disorders. Cell. 2015;162(2):375-90.

81. Satterstrom FK, et al. Large-scale exome sequencing study implicates both developmental and functional changes in the neurobiology of autism. Cell. 2020;180(3):568-84 e23.

82. Chen X, Dzakpasu R. Observed network dynamics from altering the balance between excitatory and inhibitory neurons in cultured networks. Phys Rev $E$ Stat Nonlin Soft Matter Phys. 2010;82(3 Pt 1):031907.

83. Trujillo CA, et al. Complex oscillatory waves emerging from cortical organoids model early human brain network development. Cell Stem Cell. 2019:25(4):558-69 e7.

84. Hahamy A, Behrmann M, Malach R. The idiosyncratic brain: distortion of spontaneous connectivity patterns in autism spectrum disorder. Nat Neurosci. 2015;18(2):302-9.

85. Katz LC, Shatz CJ. Synaptic activity and the construction of cortical circuits. Science. 1996;274(5290):1133-8.

86. Jimbo Y, Tateno T, Robinson HP. Simultaneous induction of pathwayspecific potentiation and depression in networks of cortical neurons. Biophys J. 1999;76(2):670-8

87. Suzuki I, Yasuda K. Detection of tetanus-induced effects in linearly lined-up micropatterned neuronal networks: application of a multi-electrode array chip combined with agarose microstructures. Biochem Biophys Res Commun. 2007;356(2):470-5.

88. Rubenstein JL. Three hypotheses for developmental defects that may underlie some forms of autism spectrum disorder. Curr Opin Neurol. 2010; 23(2):118-23.

89. Bateup HS, et al. Excitatory/inhibitory synaptic imbalance leads to hippocampal hyperexcitability in mouse models of tuberous sclerosis. Neuron. 2013;78(3):510-22.

90. Smith $\mathrm{KR}$, et al. Cadherin-10 maintains excitatory/inhibitory ratio through interactions with synaptic proteins. J Neurosci. 2017;37(46):11127-39.

91. Mossa A, et al. SHANK genes in autism: defining therapeutic targets. Prog Neuropsychopharmacol Biol Psychiatry. 2018;84(Pt B):416-23.

92. Naisbitt S, et al. Shank, a novel family of postsynaptic density proteins that binds to the NMDA receptor/PSD-95/GKAP complex and cortactin. Neuron. 1999;23(3):569-82.

93. Sala C, et al. Regulation of dendritic spine morphology and synaptic function by Shank and Homer. Neuron. 2001;31(1):115-30.

94. Gerrow K, et al. A preformed complex of postsynaptic proteins is involved in excitatory synapse development. Neuron. 2006;49(4):547-62.

95. Eltokhi A, Rappold G, Sprengel R. Distinct phenotypes of Shank2 mouse models reflect neuropsychiatric spectrum disorders of human patients with SHANK2 variants. Front Mol Neurosci. 2018;11:240.

96. Du Y, et al. Identification of a novel cortactin $\mathrm{SH} 3$ domain-binding protein and its localization to growth cones of cultured neurons. Mol Cell Biol. 1998; 18(10):5838-51.
97. Schmeisser MJ, et al. Autistic-like behaviours and hyperactivity in mice lacking ProSAP1/Shank2. Nature. 2012;486(7402):256-60.

98. Won $\mathrm{H}$, et al. Autistic-like social behaviour in Shank2-mutant mice improved by restoring NMDA receptor function. Nature. 2012;486(7402):261-5.

99. Lim CS, et al. Enhancing inhibitory synaptic function reverses spatial memory deficits in Shank2 mutant mice. Neuropharmacology. 2017;112(Pt A):104-12.

100. Wang $X$, et al. Synaptic dysfunction and abnormal behaviors in mice lacking major isoforms of Shank3. Hum Mol Genet. 2011;20(15):3093-108.

101. Lee J, et al. Shank3-mutant mice lacking exon 9 show altered excitation/ inhibition balance, enhanced rearing, and spatial memory deficit. Front Cell Neurosci. 2015;9:94,

102. Kouser $\mathrm{M}$, et al. Loss of predominant Shank3 isoforms results in hippocampus-dependent impairments in behavior and synaptic transmission. J Neurosci. 2013;33(47):18448-68.

103. Betancur C, Buxbaum JD. SHANK3 haploinsufficiency: a "common" but underdiagnosed highly penetrant monogenic cause of autism spectrum disorders. Mol Autism. 2013;4(1):17.

104. Benarroch EE. HCN channels: function and clinical implications. Neurology. 2013;80(3):304-10.

105. Jamain $\mathrm{S}$, et al. Mutations of the $\mathrm{X}$-linked genes encoding neuroligins NLGN3 and NLGN4 are associated with autism. Nat Genet. 2003;34(1):27-9.

106. Kim DS, et al. Optimizing neuronal differentiation from induced pluripotent stem cells to model ASD. Front Cell Neurosci. 2014;8:109.

107. Kirov G. CNVs in neuropsychiatric disorders. Hum Mol Genet. 2015;24(R1): R45-9.

108. Bakkaloglu B, et al. Molecular cytogenetic analysis and resequencing of contactin associated protein-like 2 in autism spectrum disorders. Am J Hum Genet. 2008;82(1):165-73.

109. Penagarikano O, et al. Absence of CNTNAP2 leads to epilepsy, neuronal migration abnormalities, and core autism-related deficits. Cell. 2011;147(1): 235-46.

110. Gao R, et al. CNTNAP2 stabilizes interneuron dendritic arbors through CASK. Mol Psychiatry. 2018;23(9):1832-50.

111. Martin-de-Saavedra, MD, et al. CNTNAP2 ectodomain, detected in neuronal and CSF sheddomes, modulates $\mathrm{Ca}^{2+}$ dynamics and network synchrony. bioRxiv, 2019.

112. Thomas AM, et al. Cntnap2 knockout rats and mice exhibit epileptiform activity and abnormal sleep-wake physiology. Sleep. 2017:40(1):Zsw026. https://doi.org/10.1093/sleep/zsw026.

113. Crino PB, Nathanson $\mathrm{KL}$, Henske EP. The tuberous sclerosis complex. N Engl J Med. 2006;355(13):1345-56.

114. Vignoli A, et al. Autism spectrum disorder in tuberous sclerosis complex: searching for risk markers. Orphanet J Rare Dis. 2015;10:154.

115. Weber AM, et al. Autism and the cerebellum: evidence from tuberous sclerosis. J Autism Dev Disord. 2000;30(6):511-7.

116. Sundberg $M$, et al. Purkinje cells derived from TSC patients display hypoexcitability and synaptic deficits associated with reduced FMRP levels and reversed by rapamycin. Mol Psychiatry. 2018;23(11):2167-83.

117. Davis GW, Bezprozvanny I. Maintaining the stability of neural function: a homeostatic hypothesis. Annu Rev Physiol. 2001:63:847-69.

118. Forrest MP, Parnell E, Penzes P. Dendritic structural plasticity and neuropsychiatric disease. Nat Rev Neurosci. 2018;19(4):215-34.

119. Santoro MR, Bray SM, Warren ST. Molecular mechanisms of fragile $X$ syndrome: a twenty-year perspective. Annu Rev Pathol. 2012;7:219-45.

120. Soden ME, Chen L. Fragile $X$ protein FMRP is required for homeostatic plasticity and regulation of synaptic strength by retinoic acid. J Neurosci. 2010;30(50):16910-21.

121. Amir RE, et al. Rett syndrome is caused by mutations in X-linked MECP2, encoding methyl-CpG-binding protein 2. Nat Genet. 1999;23(2): $185-8$.

122. Ramocki MB, et al. Autism and other neuropsychiatric symptoms are prevalent in individuals with MeCP2 duplication syndrome. Ann Neurol. 2009;66(6):771-82.

123. Blackman MP, et al. A critical and cell-autonomous role for MeCP2 in synaptic scaling up. J Neurosci. 2012;32(39):13529-36.

124. Qiu Z, et al. The Rett syndrome protein MeCP2 regulates synaptic scaling. J Neurosci. 2012;32(3):989-94.

125. Xu JC, et al. Cultured networks of excitatory projection neurons and inhibitory interneurons for studying human cortical neurotoxicity. Sci Transl Med. 2016;8(333):333ra48. 
126. Nadadhur AG, et al. Multi-level characterization of balanced inhibitoryexcitatory cortical neuron network derived from human pluripotent stem cells. PLoS One. 2017;12(6):e0178533.

127. Banerjee $A$, et al. Jointly reduced inhibition and excitation underlies circuitwide changes in cortical processing in Rett syndrome. Proc Natl Acad Sci U S A. 2016;113(46):E7287-96.

128. Amin ND, Pasca SP. Building models of brain disorders with threedimensional organoids. Neuron. 2018;100(2):389-405.

129. Bagley JA, et al. Fused cerebral organoids model interactions between brain regions. Nat Methods. 2017;14(7):743-51.

130. Quadrato $G$, et al. Cell diversity and network dynamics in photosensitive human brain organoids. Nature. 2017;545(7652):48-53.

131. Sloan SA, et al. Human astrocyte maturation captured in 3D cerebral cortical spheroids derived from pluripotent stem cells. Neuron. 2017;95(4):779-90 e6.

132. Silbereis JC, et al. The cellular and molecular landscapes of the developing human central nervous system. Neuron. 2016;89(2):248-68.

133. Splawski I, et al. Ca(M)1.2 calcium channel dysfunction causes a multisystem disorder including arrhythmia and autism. Cell. 2004;119(1):19-31.

134. Buzsaki G, Draguhn A. Neuronal oscillations in cortical networks. Science. 2004;304(5679):1926-9.

135. Uhlhaas PJ, Singer W. What do disturbances in neural synchrony tell us about autism? Biol Psychiatry. 2007:62(3):190-1.

\section{Publisher's Note}

Springer Nature remains neutral with regard to jurisdictional claims in published maps and institutional affiliations.

Ready to submit your research? Choose BMC and benefit from:

- fast, convenient online submission

- thorough peer review by experienced researchers in your field

- rapid publication on acceptance

- support for research data, including large and complex data types

- gold Open Access which fosters wider collaboration and increased citations

- maximum visibility for your research: over $100 \mathrm{M}$ website views per year

At BMC, research is always in progress.

Learn more biomedcentral.com/submissions 